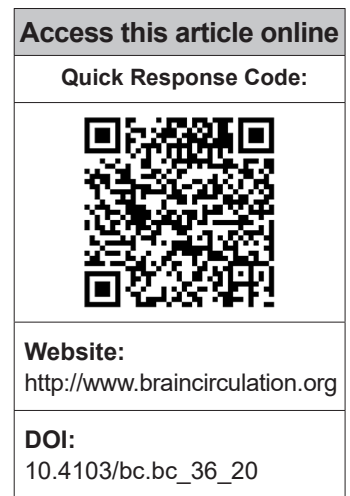

${ }^{1}$ Neuroscience Center of Excellence, School of Medicine, Louisiana State University Health Sciences Center, New Orleans, ${ }^{2}$ Department of Pediatrics, School of Medicine, University of California, Irvine,

${ }^{4}$ Department of Chemistry, University of Southern California, Los Angeles,

CA, ${ }^{3}$ Department of

Morphology and Institute of Biomedicine, School of Medicine, Laboratory of the Biology of Tissue

Healing, Ontogeny and Nutrition, Federal

University of Ceara, Fortaleza, Brazil

Address for correspondence: Dr. Ludmila Belayev, Neuroscience Center of Excellence, School of

Medicine, Louisiana State University Health New Orleans, 2020 Gravier Street, Suite D, New Orleans, LA, 70112, USA. E-mail: Ibelay@Isuhsc.edu Dr. Nicolas G. Bazan, Neuroscience Center of Excellence, School of Medicine, Louisiana State University Health New Orleans, 2020 Gravier Street, Suite

D, New Orleans, LA, 70112, USA.

E-mail: nbazan@Isuhsc. edu

Submission: 17-08-2020

Revised: 10-09-2020

Accepted: 18-10-2020

Published: 29-12-2020

\title{
Blocking pro-inflammatory
} platelet-activating factor receptors and activating cell survival pathways: A novel therapeutic strategy in experimental ischemic stroke

\author{
Ludmila Belayev ${ }^{1}$, Andre Obenaus ${ }^{2}$, Pranab K. Mukherjee ${ }^{1}$, Eric J. Knott', \\ Larissa Khoutorova ${ }^{1}$, Madigan M. Reid ${ }^{1}$, Cassia R. Roque ${ }^{1,3}$, Lawrence Nguyen ${ }^{2}$, \\ Jeong Bin Lee ${ }^{2}$, Nicos A. Petasis', Reinaldo B. Oria ${ }^{1,3}$, Nicolas G. Bazan ${ }^{1}$
}

\section{Abstract:}

OBJECTIVE: Acute ischemic stroke triggers complex neurovascular, neuroinflammatory, and synaptic alterations. This study explores whether blocking pro-inflammatory platelet-activating factor receptor (PAF-R) plus selected docosanoids after middle cerebral artery occlusion (MCAo) would lead to neurological recovery. The following small molecules were investigated: (a) LAU-0901, a PAF-R antagonist that blocks pro-inflammatory signaling; and (b) derivatives of docosahexaenoic acid (DHA), neuroprotectin D1 (NPD1), and aspirin-triggered NPD1 (AT-NPD1), which activates cell survival pathways and are exert potent anti-inflammatory activity in the brain.

MATERIALS AND METHODS: Sprague-Dawley rats received $2 \mathrm{~h}$ MCAo and LAU-0901 (30 or $60 \mathrm{mg} / \mathrm{kg}, 2 \mathrm{~h}$ after stroke), NPD1, and AT-NPD1 (333 $\mu \mathrm{g} / \mathrm{kg})$, DHA $(5 \mathrm{mg} / \mathrm{kg})$, and their combination were administered intravenous at $3 \mathrm{~h}$ after stroke. Behavior testing and ex vivo magnetic resonance imaging were conducted on day 3 or 14 to assess lesion characteristics and lipidomic analysis on day 1. Series 1 (LAU-0901 + NPD1, 14d), Series 2 (LAU-0901 + AT-NPD1, 3d), and Series 3 (LAU-0901 + DHA, 1d). RESULTS: All combinatory groups improved behavior compared to NPD1, AT-NPD1, or DHA treatments alone. Total lesion volumes were reduced with LAU-0901 + NPD1 by $62 \%$ and LAU-0901 + AT-NPD1 by $90 \%$ treatments versus vehicle groups. LAU-0901 and LAU-0901 + DHA increased the production of vasoactive lipid mediators (prostaglandins: $\mathrm{PGE}_{2}, \mathrm{PGF}_{2-\alpha}, 6-\mathrm{keto}^{-\mathrm{PGF}_{1-\alpha}}$, and $\mathrm{PGD}_{2}$ ) as well an inflammatory regulating mediator hydroxyoctadecadienoic acid. In contrast, LAU-0901 and LAU-0901 + DHA decreased the production of 12-hydroxyeicosatetraenoic acid, a pro-inflammatory mediator.

CONCLUSION: Combination therapy with LAU-0901 and selected docosanoids is more effective than the single therapy, affording synergistic neuroprotection, with restored pro-homeostatic lipid mediators and improved neurological recovery. Altogether, our findings support the combinatory therapy as the basis for future therapeutics for ischemic stroke.

Keywords:

Docosanoids, ischemia/reperfusion, magnetic resonance imaging, neuroinflammation, platelet-activating factor-receptors antagonist

This is an open access journal, and articles are distributed under the terms of the Creative Commons Attribution-NonCommercial-ShareAlike 4.0 License, which allows others to remix, tweak, and build upon the work non-commercially, as long as appropriate credit is given and the new creations are licensed under the identical terms.

Forreprintscontact:WKHLRPMedknow_reprints@wolterskluwer.com
How to cite this article: Belayev L, Obenaus $A$, Mukherjee PK, Knott EJ, Khoutorova L, Reid MM, et al. Blocking pro-inflammatory platelet-activating factor receptors and activating cell survival pathways: A novel therapeutic strategy in experimental ischemic stroke. Brain Circ 2020;6:260-8. 


\section{Introduction}

Tschemic stroke is a leading cause of death and the L primary cause of permanent disability. Current therapeutic options are limited and do not address the associated immune response and acute inflammation that occur at the site of cerebral ischemia. Ischemia-reperfusion injury is associated with dysregulation of multiple neuroinflammatory pathways ${ }^{[1,2]}$ and disruption of neuronal circuits, which, in turn, results in many of the pathologies affecting stroke survivors. ${ }^{[3]}$ We are introducing a novel approach to pharmacologically target and resolve neuroinflammatory disruptions to attain sustained neuronal network integrity and neurologic recovery. To address the multifaceted pathogenic components of stroke, we proposed a combination therapy to block pro-inflammatory events ${ }^{[1,4]}$ while simultaneously promoting neuroprotection and inflammation resolution. ${ }^{[4,5]}$

A robust suppression of pro-inflammatory signaling has been achieved by the use of LAU-0901 (2,4,6-trimethyl-1, 4-dihydro-pyridine-3, 5-dicarboxylic acid), a small molecule antagonist of the platelet-activating factor receptor (PAF-R). ${ }^{[6]}$ Platelet-activating factor (PAF; 1-Oalkyl-2-acyl-sn-3-phosphocholine) release may lead to substantial cerebral injury by enhancing intracellular calcium, disrupting the blood-brain barrier, reducing cerebral blood flow, and activating leukocytes. ${ }^{[6]}$ Excessive activated immune cell PAF release triggers neuronal injury, and inhibition of this process plays a critical role in a neuronal survival and prevention of ischemic brain injury. ${ }^{[7,8]}$ LAU-0901, a highly potent and selective PAF-R antagonist, has been examined in models of focal cerebral ischemia in rats and mice. ${ }^{[9]}$ LAU-0901 improved behavioral deficits and reduced infarct volumes when administered $2 \mathrm{~h}$ after middle cerebral artery occlusion (MCAo). ${ }^{[9,10]}$ In addition, LAU-0901 conferred enduring neuroprotection in animals allowed to survive for several weeks after stroke. ${ }^{[11]}$

Neuroprotective effects and hastening of inflammatory resolution have been achieved by administration of bioactive docosanoids, endogenous lipid mediators derived from docosahexaenoic acid (DHA), a major omega-3 fatty acid in the brain following brain ischemia-reperfusion. Neuroprotectin D1 (NPD1) and aspirin-triggered NPD1 are synthetic docosanoids that have been previously studied by our group. They have been found to promote neuronal cell survival with important anti-inflammatory activity. ${ }^{[12-14]}$ The biological activity of NPD1 and AT-NPD1 has been attributed to specific activation or modulation of signaling pathways associated with the immune system, inflammation, cell survival, and/or cell-cell interactions. ${ }^{[5,14]}$
Increasing evidence suggests that resolution of inflammation is not a passive process due to exhaustion of the signaling per se, but rather an orchestrated interaction of a large number of mediators, which actively suppress the inflammatory response. ${ }^{[2]}$ Major steps in the process include the removal of dead cells, the development of an anti-inflammatory environment, and the generation of prosurvival factors fostering tissue reconstruction and repair. ${ }^{[2]}$ The modulation of eicosanoid pathways and lipid mediators has been addressed by combining LAU-0901 with DHA, which activates cell survival pathways with anti-inflammatory and neuroprotective activity in the brain. ${ }^{[15]}$

Therefore, in this study, the therapeutic efficacy of LAU-0901 combinatory therapy with selected docosanoids (DHA, NPD1, and AT-NPD1) was investigated using a well-established rat model of transient ischemia, ${ }^{[16]}$ with the aid of multimodal magnetic resonance imaging (MRI), neurobehavioral assays, and lipidomic analysis. No prior studies have used this novel combinatory approach for the treatment of ischemic stroke.

\section{Materials and Methods}

\section{Animals}

All studies were approved by the Institutional Animal Care and Use Committee of the Louisiana State University Health Sciences Center New Orleans. Male Sprague-Dawley rats (280-370 g) obtained from Charles River Laboratories (Wilmington, MA) were used in all studies. Anesthesia was induced with 3\% isoflurane in a mixture of $70 \%$ nitrous oxide and $30 \%$ oxygen. All rats were orally intubated and mechanically ventilated. The catheters were implanted into the right femoral artery and vein for the blood sampling and infusion of drugs. Serial analyses of arterial blood gases, plasma glucose, hematocrit, and arterial blood pressure were conducted before and during a surgical procedure. Rectal (CMA/150 Temperature Controller, CMA/Microdialysis AB, Stockholm, Sweden) and cranial (temporalis muscle; Omega Engineering, Stamford, CT) temperatures were maintained at $36^{\circ} \mathrm{C}$ to $37^{\circ} \mathrm{C}$ before, during, and after MCAo. Rectal temperature and body weight were monitored daily during the survival period.

\section{In vivo ischemia model: Transient middle cerebral artery occlusion}

Rats underwent $2 \mathrm{~h}$ of right MCAo by an intraluminal filament, as previously described. ${ }^{[16]}$ A 3-0 monofilament coated with poly-L-lysine was advanced through the external carotid artery to the MCA until mild resistance was felt. Animals were allowed to awaken from anesthesia and at $60 \mathrm{~min}$ of MCAo were tested on a standardized neurobehavioral battery to confirm the 
presence of a high-grade neurological deficit. ${ }^{[16]}$ After $2 \mathrm{~h}$ of MCAo, rats were reanesthetized with the same anesthetic combination. Temperature probes were reinserted, intraluminal sutures were carefully removed, and the animals were allowed to survive for $1,3,7$, or 14 days according to experimental design with free access to food and water.

Treatments and experimental protocols

Rats were randomly assigned to LAU-0901 (IP, 30 or $60 \mathrm{mg} / \mathrm{kg}$, at $2 \mathrm{~h}$ after onset of stroke), (NPD1; $333 \mu \mathrm{g} / \mathrm{kg}$ ), aspirin-triggered (AT-NPD; $333 \mu \mathrm{g} / \mathrm{kg}$ ), docosahexanoic acid (DHA; $5 \mathrm{mg} / \mathrm{kg})$ or vehicle $(0.9 \%$ saline) treatment groups. NPD1, AT-NPD1, and DHA were administered intravenously $3 \mathrm{~h}$ after the onset of MCAo at a constant rate over 3 min using an infusion pump. All treatments were administered by researchers blinded to the treatment groups.

Three study protocols were applied: Series 1, Effect of LAU-0901 + NPD1: LAU-0901 (30 mg/kg) and NPD1 alone or in combination; behavior was evaluated on days 1, 2, 3,7 , and 14 , followed by ex vivo MRI on day 14 . Series 2, effect of LAU-0901 + AT-NPD1: LAU-0901 (60 mg/kg) and AT-NPD1 alone or in combination; behavior was evaluated on days 1,2 , and 3 , followed by ex vivo MRI on day 3. Series 3, effect of LAU-0901 + DHA: LAU-0901 (60 mg/kg) and DHA alone or in combination; behavior was evaluated at $4 \mathrm{~h}$ and day 1 , followed by lipidomic analyses on day 1 .

\section{Behavioral assessments}

The primary behavioral end point was the composite neurological score $(0-12$ points, $0=$ normal, and 12 = maximal deficit) as we previously described.$^{[16]}$ The battery consisted of two tests to assess various aspects of neurologic function: (1) the postural reflex test, to examine upper body posture while the animal is suspended by the tail and (2) the forelimb placing test, to examine sensorimotor integration in forelimb placing responses to visual, tactile, and proprioceptive stimuli. Rats that did not demonstrate high-grade contralateral deficit (score, 10-11) were excluded from further study. Tests were performed by an observer blinded to the treatment groups at $60 \mathrm{~min}$ (during MCAo) and then on days 1, 2, 3, 7, or 14 after MCAo.

Magnetic resonance imaging acquisition and analysis of total lesion, core, and penumbra volumes

High-resolution ex vivo MRI was performed on $4 \%$ paraformaldehyde-fixed brains at day 3 or 14 using an 11.7T Bruker Advance $8.9 \mathrm{~cm}$ horizontal bore instrument equipped with an $89 \mathrm{~mm}$ (ID) receiver coil (Bruker Biospin, Billerica, MA). T2-weighted image (T2WI) and T2 relaxation maps were computed as we previously described. ${ }^{[17]}$ We used hierarchical region splitting (HRS) to automatically identify core and penumbra volumes (total lesion $=$ core + penumbra) from T2 relaxation maps. ${ }^{[17]}$ Our core and penumbral tissue determination using HRS (implemented in Matlab) was validated previously by the use of perfusion-weighted imaging/diffusion-weighted imaging subtractions at each brain level. ${ }^{[17]}$ The penumbra from HRS was defined using T2 values (ms), which were between normal-appearing brain tissue and ischemic core. Data from each modality were summarized per group.

\section{Brain sampling and lipidomic analysis}

Rats were sacrificed on day 1 to investigate the effect of LAU-0901 plus DHA on the expression of different lipid mediators in the ischemic core and penumbra. The brains were removed, divided into right and left hemispheres, and the penumbra (cortex) and core (subcortex) were dissected at bregma level - 0.3 $\mathrm{mm}$ for lipidomic analysis. The following lipids were extracted: prostaglandins $\left(\mathrm{PGE}_{2}, \mathrm{PGF}_{2-}{ }^{\alpha}, 6\right.$-keto- $\mathrm{PGF}_{1-}{ }^{\alpha}$, $\mathrm{PGD}_{2}$ ), hydroxyoctadecadienoic acid (HODE), 11-dehydro-thromboxane B2, thromboxane B2 (TXB2), 12-hydroxyeicosatetraenoic acid (12-HETE). They were characterized by liquid chromatography-photodiode array detection-electrospray ionization-tandem mass spectrometry (MS/MS) using a TSQ quantum ultra performance liquid chromatography-MS/MS triple stage tandem mass spectrometer from Thermo Electronic Company, Inc., at SRM mode, and results were normalized to total sample protein. ${ }^{[13]}$

\section{Statistical analysis}

Values are reported as means \pm standard error of the mean two-tailed Student's $t$-test were used for two-group comparisons. For multiple group comparisons, repeated measures analysis of variance, followed by Bonferroni tests, were performed. A value of $P<0.05$ was regarded as statistically significant.

\section{Results}

\section{Physiological variables and mortality}

Rectal and cranial (temporalis muscle) temperatures, arterial blood gases, and plasma glucose showed no significant differences between all groups. There were no adverse behavioral side effects observed after LAU-0901, NPD1, AT-NPD1, or DHA administration in all groups. No animals died during this study.

LAU-0901+neuroprotectin D1 improved behavioral function and attenuated ischemic brain damage 14 days after middle cerebral artery occlusion

We investigated whether treatments with LAU-0901 and NPD1 alone as well as combination treatment with 
LAU-0901 + NPD1 affect behavior and infarct size after brain ischemia 14 days posttherapy. Experimental design presented in Figure 1a. LAU-0901 and NPD1 treatments alone improved behavioral scores beginning on day 1 by $16 \%$ and $37 \%$, which persisted through day 14 by $26 \%$ and $40 \%$ compared to vehicle group [Figure $1 \mathrm{~b}$ ]. The neuroprotective effect was enhanced using the LAU-0901 + NPD1, which resulted in improved behavioral scores up to $54 \%$ on day 14 [Figure $1 \mathrm{~b}$ ]. Behavioral score was significantly different between LAU-0901 + NPD1 and LAU-0901, but not between LAU-0901 + NPD1 and NPD1 treatments alone. Combinatory and NPD1 - alone treatments remarkably improved time courses for recovery of visual sideways, tactile dorsal, and lateral contralateral forelimb reactions at days 2-14 [Figure 1c-e].

Ischemic core, penumbra, and total lesion volumes computed from T2 maps on day 14 presented in Figure 2. T2WI revealed large lesions and T2 hyperintensities, which were observed in the ischemic core and penumbra of vehicle-treated rats, consistent with edema formation. In contrast, all treatments, especially NPD1 and
LAU-0901 + NPD1, had smaller lesion volumes that were visible only in a small portion of the cortex and subcortical areas. Three-dimensional lesion volumes were dramatically reduced by NPD1 and LAU-0901 + NPD1 treatments compared to vehicle group [Figure 2b]. Quantitative total, core, and penumbra T2WI lesion volumes were significantly reduced by all treatments compared to vehicle-treated group [Figure 2c]. Combinatory treatment was significantly different from LAU-0901 treatment alone on the reduction of lesions in the ischemic core [Figure 2c].

LAU-0901 + AT-neuroprotectin D1 provided superior neuroprotection 3 days after middle cerebral artery occlusion

Experimental design is presented in Figure $3 a$. Treatments with LAU-0901 and AT-NPD1 alone improved total neurological score beginning on day 1 , which persisted through day 3 by $33 \%$ and $27 \%$ compared to vehicle group [Figure $3 \mathrm{~b}$ ]. Combinatory treatment with LAU-0901 + AT-NPD1 improved behavioral score up to $54 \%$ on day 3 [Figure $3 \mathrm{~b}$ ]. Total lesion volumes (computed from T2 maps) were

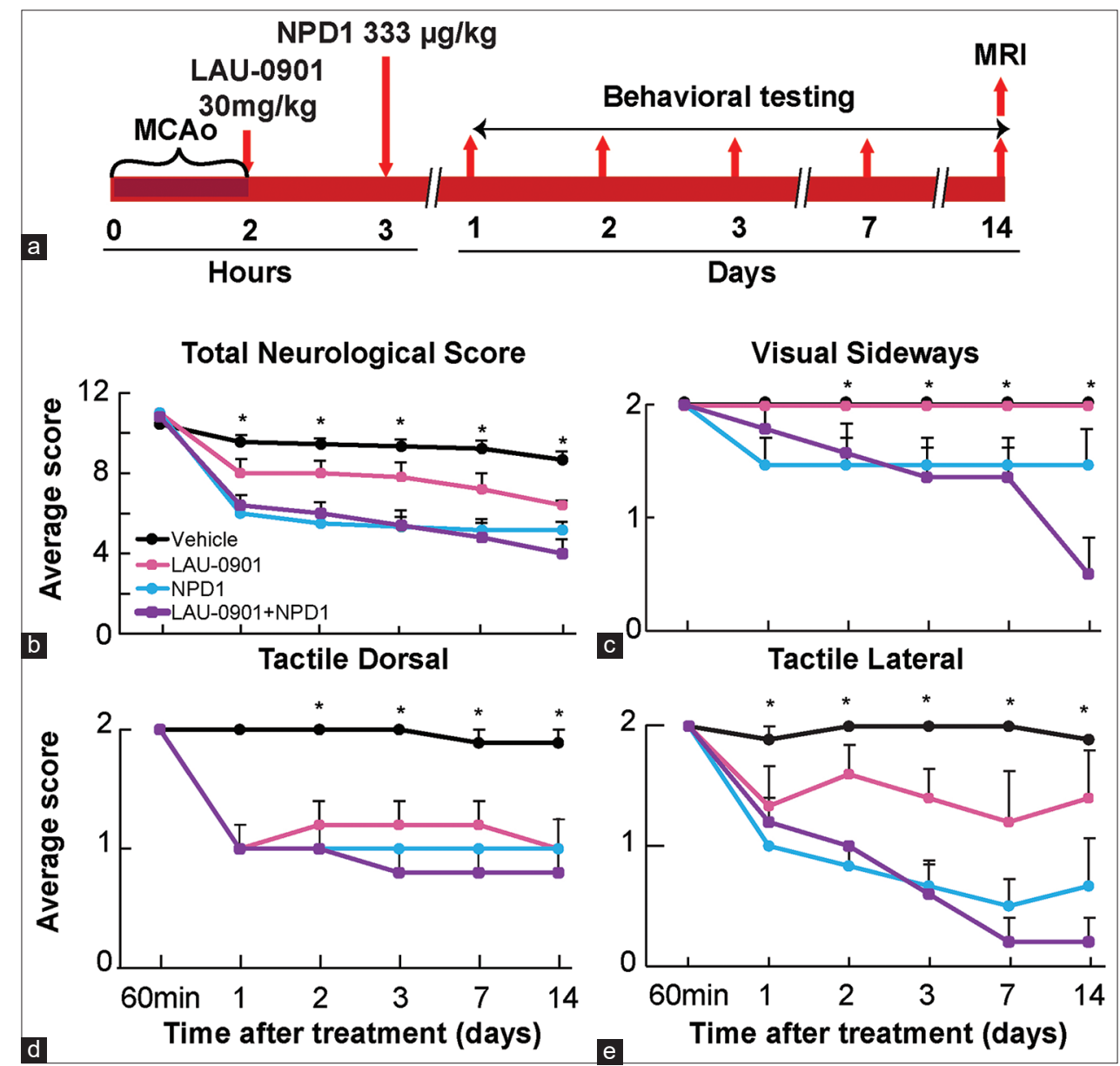

Figure 1: LAU-0901 plus neuroprotectin D1 improves behavior 14 days after stroke. (a) Experimental design. (b) total neurological score (normal $=0, \max =12)$ and (c-e) time course of recovery forelimb reactions. $P<0.05$, *Vehicle versus all treatments; $n=5-6 /$ group 


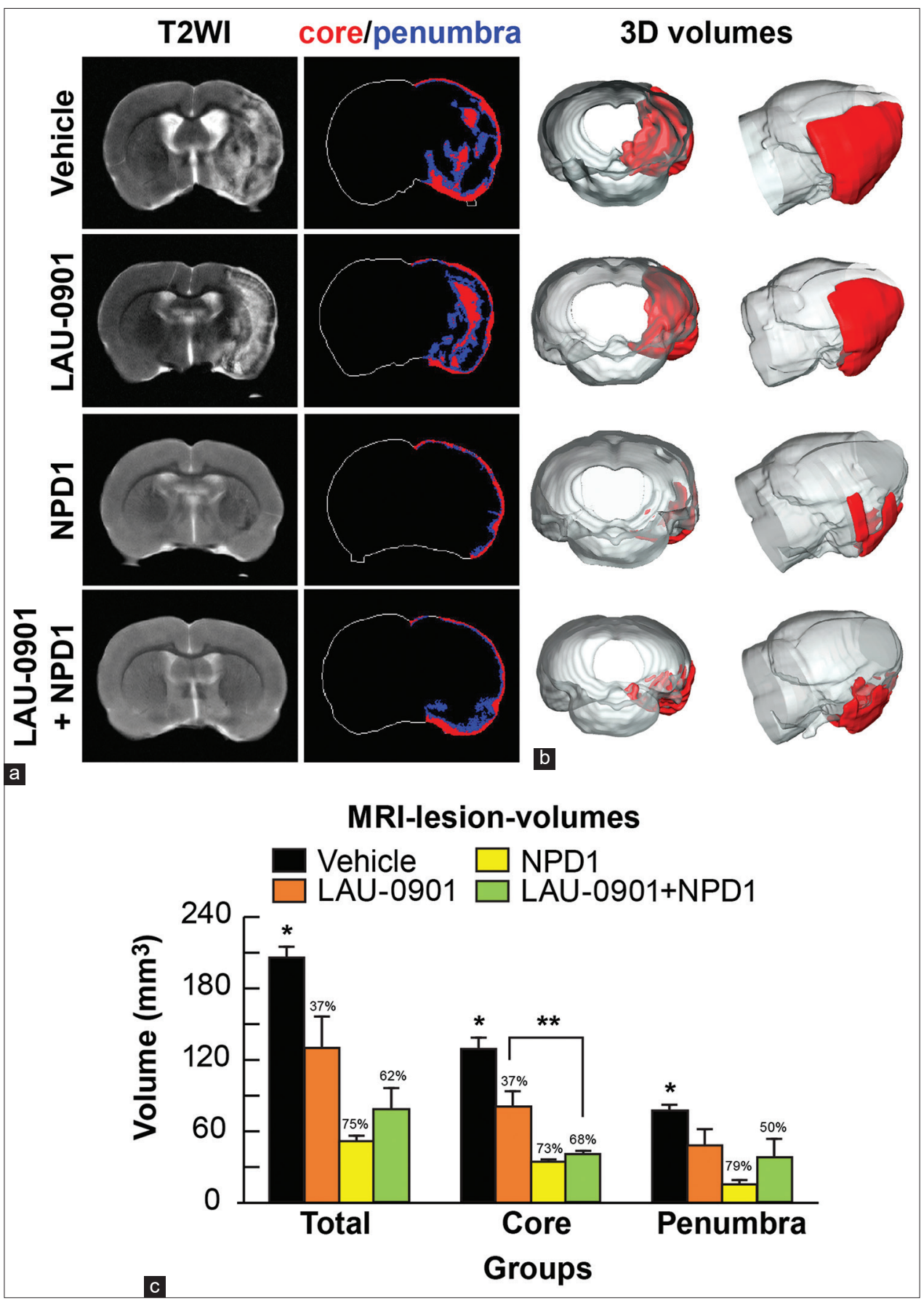

Figure 2: LAU-0901 plus neuroprotectin D1 reduces magnetic resonance imaging -derived stroke lesion volumes. (a) Representative T2-weighted image, core/penumbra, and (b) Three-dimensional lesion volumes computed from T2 maps on day 14. Core (red) and penumbral (blue) tissues were automatically identified. (c) Ischemic core, penumbra, and total lesion volumes. $P<0.05$, *Vehicle versus all treatments; **LAU-0901 versus LAU-0901+ neuroprotectin D1, $n=5-6 /$ group

reduced by all treatments [Figure 3c]. Combinatory treatment reduced total lesion volume compared to LAU-0901-alone group [Figure 3c]. Representative T2WI, pseudo-colored images, and 3D volumes illustrate that all treatments reduced lesion in ischemic core and penumbra compared to vehicle [Figure 3d-f]. Lesions were dramatically reduced by combinatory treatment with LAU-0901 + NPD1 and were primarily restricted to subcortical areas.
LAU-0901 + docosahexaenoic acid modulates regulation of cerebral blood flow and inflammatory signaling, which leads to neurological recovery 1 day after middle cerebral artery occlusion We investigated the effect of treatment with LAU-0901 and DHA alone and in combination on behavior as well as the expression of different lipid mediators 1 day after ischemic stroke. Experimental design presented 


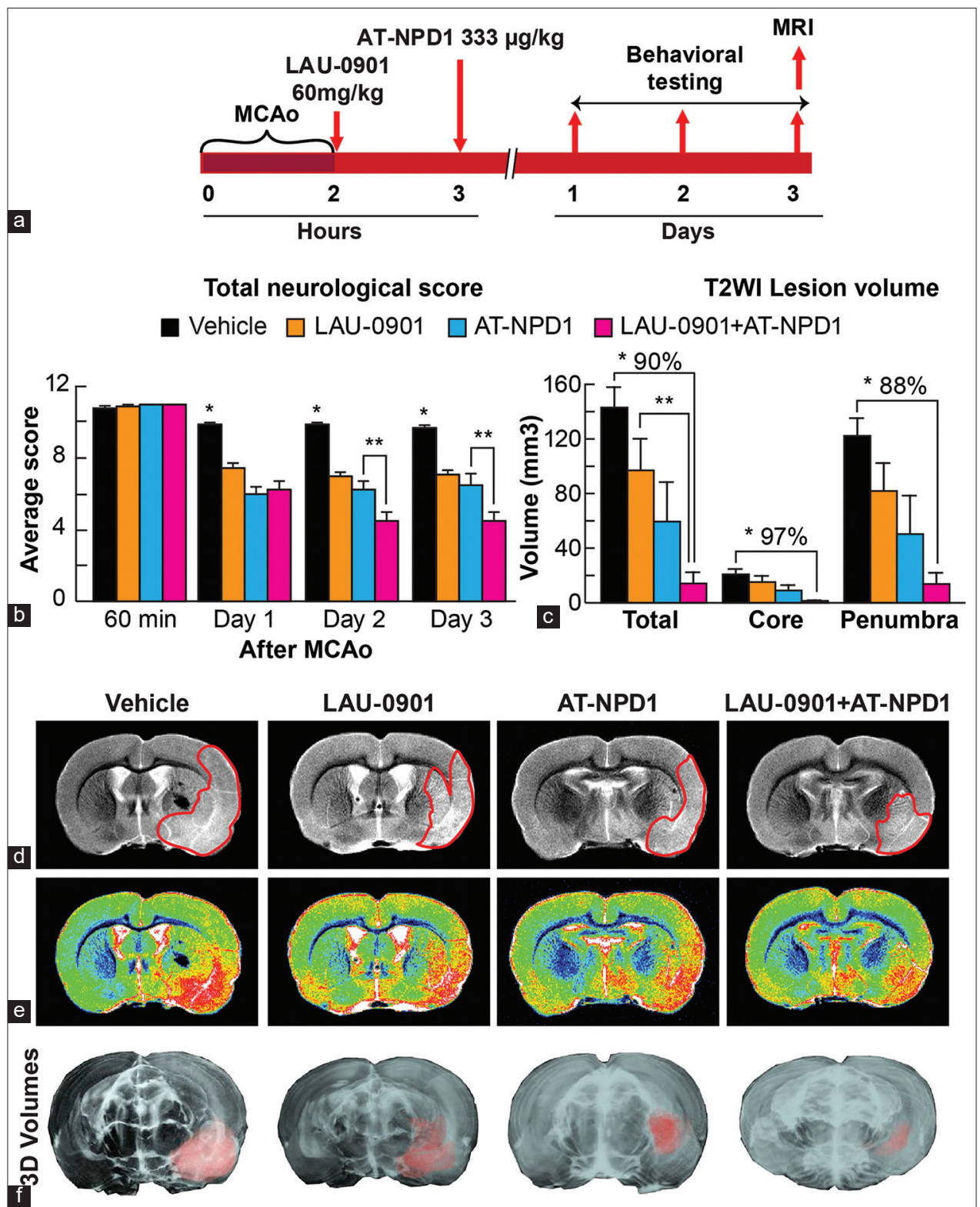

Figure 3: LAU-0901 plus aspirin-triggered neuroprotectin D1 is protective 3 days after stroke. (a) experimental design. (b) total neurological score (normal $=0$, max $=12$ ). (c) Total, core, and penumbra lesion volumes computed from T2 maps. (d) Representative T2-weighted image, (e) pseudo-colored T2 images, and (f) collapsed 3D lesion volumes (red) superimposed on T2-weighted image. $P<0.05$, ${ }^{*}$ Vehicle versus all treatments; ${ }^{*}$ LAU-0901+ neuroprotectin D1 versus aspirin-triggered neuroprotectin D1 or LAU-0901, $n=5-7 /$ group

in Figure 4a. LAU-0901 and DHA treatments alone improved behavioral scores compared to vehicle groups by $30 \%-35 \%$ [Figure $4 \mathrm{~b}$ ]. The neuroprotective effect was enhanced using the LAU-0901 + DHA by $47 \%$ on day 1 .

Expression of prostaglandins $\left(\mathrm{PGE}_{2}, \mathrm{PGF}_{2-\alpha}\right.$, 6-keto-PGF ${ }_{1-\alpha^{\prime}}$ and $\mathrm{PGD}_{2}$ ), HODE, and 12-HETE from the ipsilateral ischemic core and penumbra are presented in Figure 4c-h. The highest upregulation was observed in penumbra regions by LAU-0901 alone and in combination with DHA. The appearance of prostaglandins when treated with LAU-0901 alone increased 18-fold for $\mathrm{PGE}_{2}, 47$-fold for $\mathrm{PGF}_{2-\alpha,}, 10$ fold for

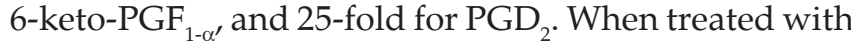
LAU-0901 + DHA, an increase 6-fold for PGE, 13-fold for $\mathrm{PGF}_{2-\alpha^{\prime}}$ 6-fold for 6-keto-PGF ${ }_{1-\alpha^{\prime}}$ and 14-fold for PGD was observed compared to vehicle [Figure 4c-f]. HODE expression increased 5-fold with LAU-0901 treatment alone and 4-fold with LAU-0910 + DHA [Figure 4g]. Expression of 12-HETE was reduced by DHA, LAU-0901, and LAU-0901 + DHA (83, 67, 72\%, respectively) [Figure $4 \mathrm{~h}$ ].

\section{Discussion}

In this study, we examined the therapeutic efficacy of LAU-0901, a PAF-R antagonist, in combination with 


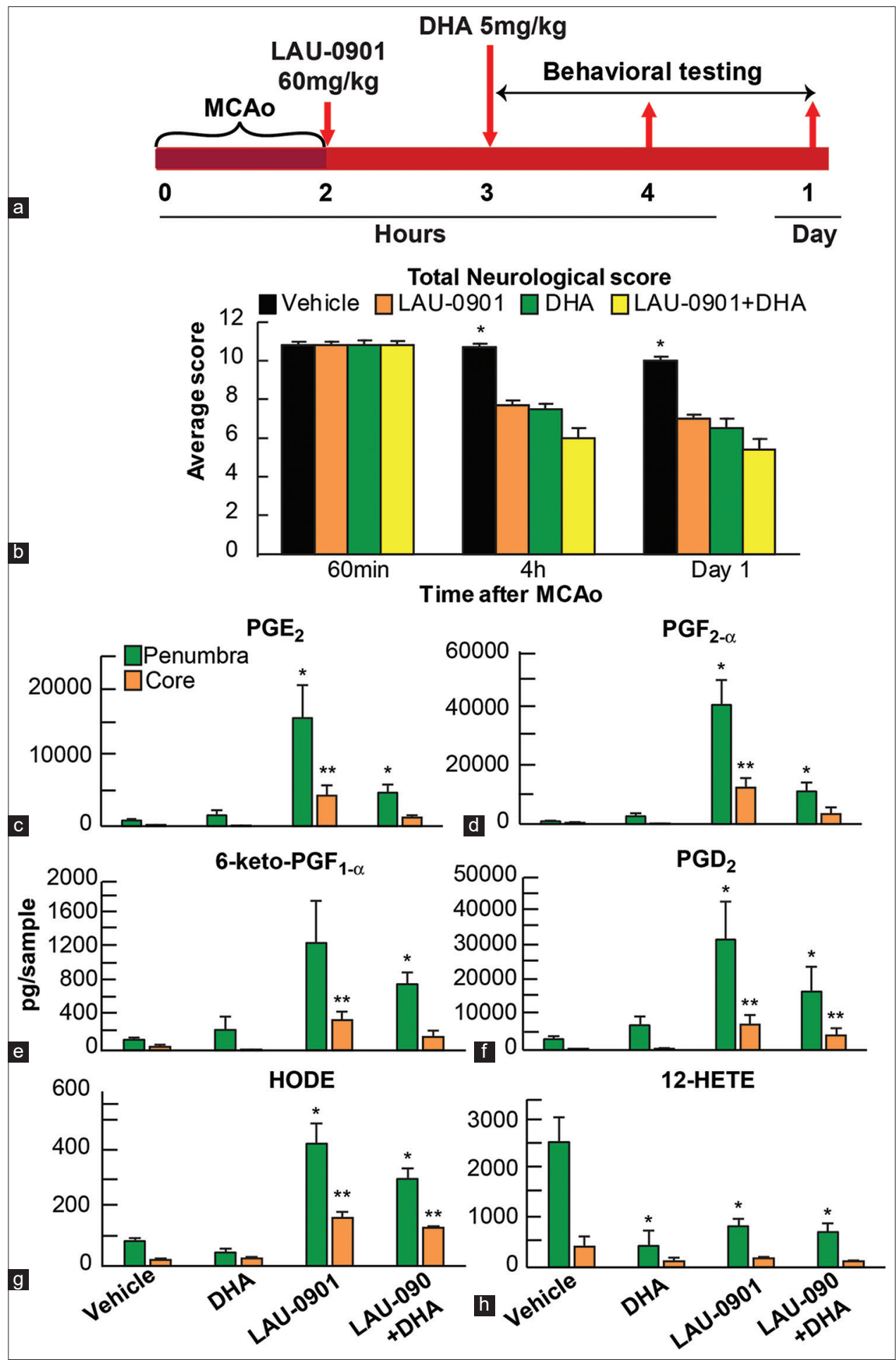

Figure 4: LAU-0901 plus DHA improves behavior and modulates inflammatory response 1 day after stroke. (a) Experimental design. (b) Total neurological score.(c-h). Quantitative analysis of lipid extracts after middle cerebral artery occlusion from the ischemic core and penumbra. $P<0.05,{ }^{*} A l l$ treatments versus vehicle (penumbra); ${ }^{* *} A l l$ treatments versus vehicle (core), $n=3-4 /$ group

NPD1, AT-NPD1, and DHA following MCAo in rats. We have shown here for the first time that LAU-0901 in combination with the various docosanoids reduced ischemic brain injury, promoted functional recovery, and restored our pro-homeostatic lipid mediator pathway after experimental ischemic stroke.
Our results demonstrate that LAU-0901 in combination with NPD1 and AT-NPD1 significantly reduced infarct volume and improved neurological score when compared to LAU-0901 alone. This indicates the superior therapeutic efficacy of combinatory treatment following ischemic stroke. LAU-0901 was used to 
inhibit pro-inflammatory signaling by PAF, which accumulates in response to cell injury including cerebral ischemia-reperfusion. During ischemia, PAF increases and then becomes a pro-inflammatory messenger as well as a mediator of neurotoxicity that drives neuronal damage. ${ }^{[10,18]}$ We have previously shown that LAU-0901 provides robust neuroprotection when used in experimental ischemic stroke, reducing the volume of cerebral infarction and improving neurological scores. ${ }^{[10]}$

NPD1 is DHA-derived lipid mediator, which promotes cell survival under uncompensated oxidative stress, an early event during neuronal cell death. ${ }^{[19]}$ Enzymatic lipoxygenation of DHA in experimental ischemic stroke results in NPD1 formation, which then counteracts pro-inflammatory bioactivity and contributes to sustained neuroprotection. ${ }^{[2,21]}$ NPD1 is protective following ischemic stroke by reducing infarct volume, improving neurological score, and has been shown to attenuate polymorphonuclear leukocyte infiltration, inhibit ischemia induction by cyclooxygenase- 2 and nuclear factor $\kappa B$ activation. ${ }^{[21-23]}$ AT-NPD1 is a novel endogenous DHA-derived lipid mediator generated through aspirin-acetylated COX-2. When delivered at $3 \mathrm{~h}$ after stroke onset, it provides sustained neurobehavioral recovery, reduces brain infarction and brain edema, improves tissue matrix, and protects white matter. ${ }^{[13]}$ Both combinatory treatment with LAU-0901 + NPD1 and LAU-0901 + AT-NPD1 improved neurological recovery better than any of the treatments alone, supporting the therapeutic efficacy of their synergistic neuroprotection.

Combinatory treatment with LAU-0901 + DHA and LAU-0901 alone elicited an increased expression of prostaglandins, $\mathrm{PGE}_{2}, \mathrm{PGF}_{2-\alpha^{\prime}}$ 6-keto- $\mathrm{PGF}_{1-\alpha^{\prime}}$ and $\mathrm{PGD}_{2}$, compared to vehicle animals. Prostaglandins are bioactive lipids derived from the metabolism of arachidonic acid. They sustain homeostatic functions and mediate pathogenic mechanisms including inflammatory response. ${ }^{[24,25]}$ The increased expression of these metabolites indicates that the tissue maintained its ability to regulate cerebral blood flow and modulate the inflammatory process, which previous studies have shown to improve functional outcomes following stroke. ${ }^{[2,27]}$ Cerebral ischemia initiates a cascade of events that stimulates the release of free fatty acids from cell membranes, particularly arachidonic acid and DHA. These bioactive lipid messengers, in turn, give rise to several downstream lipid messengers such as PAF and eicosanoids (prostaglandins and leukotrienes). These molecules may have either neuroprotective or neurodegenerative effects on post-stroke brain tissue; therefore, they largely contribute to the outcome and recovery from stroke.

$\mathrm{PGE}_{2^{\prime}}, \mathrm{PGF}_{2-\alpha^{\prime}}$ 6-keto-PGF ${ }_{1-\alpha^{\prime}}$ and $\mathrm{PGD}_{2}$ all exhibit vasoactive bioactivity and have been shown to improve poststroke outcomes. $\mathrm{PGE}_{2}$ exerts a variety of functions by binding primarily to fourmembrane receptors known as E-prostanoid $1,2,3$, and 4 having varied effects physiologically. ${ }^{[28]}$ Genetic knockout of the EP2 receptor has been shown to significantly increase lesion volume at $24 \mathrm{~h}$ in mice subjected to cerebral ischemia, and the activation of this receptor has been shown to protect against excitotoxic and anoxic injury following MCAo. ${ }^{[28,29]}$ 6-keto- $\mathrm{PGF}_{1-\alpha}$ is a metabolite derived from prostacyclin; it has been shown that targeting the prostaglandin $\mathrm{I}_{2}$ prostanoid receptor following MCAo reduces cerebral ischemic injury and promotes long-term neurological recovery following ischemic stroke. ${ }^{[30]} \mathrm{PGD}_{2}$ is the most abundant prostaglandin in the brain and acts as a modulator of the neuroinflammatory process. $\mathrm{PGD}_{2}$ acts primarily on the DP1 receptors, which have a beneficial effect on stroke outcomes when activated, minimizing excitotoxic and ischemic brain damage. ${ }^{[27,29]}$

An increase in the expression of HODE in rats treated with LAU-0901 and LAU-0901 + DHA, as well as a decrease in the expression of 12-HETE was also observed in our study. HODE, a derivative of linoleic acid, is thought to be involved in the regulation of neurotransmission and inflammation in response to brain injury, reducing glutamate excitotoxicity. ${ }^{[31,32]}$ 12-HETE, a derivative of arachidonic acid, is a bioactive lipid formed by the action of 12-lipoxygenase enzyme, which is upregulated in hypoxic conditions. 12-HETE is a pro-inflammatory lipid mediator, which can further ischemic damage due to increased inflammatory signaling and cell death. Inhibition of 12-HETE has previously been shown to reduce infarct size and improve behavioral parameters. ${ }^{[33]}$ Our results indicate that treatment with LAU-0901 regulates the presence of metabolites that act to reduce inflammation and excitotoxicity-driven neuronal damage. The presence of markers observed in rats treated with LAU-0901 and LAU-0901 + DHA is likely the result of modulating perfusion by increasing cerebral blood flow through vasoactive prostaglandins, as well as suppression of pro-inflammatory signaling through the decreased expression of 12-HETE and increased expression of HODE, as well as the action of LAU-0901 inhibiting PAF.

\section{Conclusion}

Altogether our findings support our prediction that therapeutic efficacy would be enhanced using a combination of a PAF inhibitor, LAU-0901, with selected docosanoids, as well as providing synergistic neuroprotection in the postischemic brain. This compound therapy restored pro-homeostatic lipid mediators with improved neurological recovery and may hold promise for future therapeutics development against ischemic stroke.

\section{Acknowledgments}

We would like to thank Brazilian 
CAPES (88881.311939/2018-01) for supporting PhD student (Cassia R. Roque). We acknowledge the assistance of Mr. Saburi Eliamani at the Research Imaging Center at Loma Linda University for acquisition of the MRI data.

\section{Financial support and sponsorship}

This study was supported by the National Institute of Neurological Disorders and Stroke under award numbers 1R01NS109221 (N.G.B. and L.B.) and 1R01NS104117 (N.G.B. and L.B.).

\section{Conflicts of interest}

There are no conflicts of interest.

\section{References}

1. Bazan NG, Halabi A, Ertel M, Petasis NA. Neuroinflammation. In: Brady ST, Siegel GJ, Albers RW, Price DL, editors. Basic Neurochemistry. $8^{\text {th }}$ ed.., Ch. 34. New York: Academic Press; 2012. p. 610-20. Available from: http:/ / www.sciencedirect.com/ science/article/pii/B9780123749475000341. [Last accessed on $2020 \mathrm{Jul} 23]$

2. Iadecola C, Anrather J. The immunology of stroke: From mechanisms to translation. Nat Med 2011;17:796-808.

3. Bazan NG. Omega-3 fatty acids, pro-inflammatory signaling and neuroprotection. Curr Opin Clin Nutr Metab Care 2007;10:136-41.

4. Serhan CN, Yacoubian S, Yang R. Anti-inflammatory and proresolving lipid mediators. Annu Rev Pathol 2008;3:279-312.

5. Serhan CN, Petasis NA. Resolvins and protectins in inflammation resolution. Chem Rev 2011;111:5922-43.

6. Bazan NG. Lipid signaling in neural plasticity, brain repair, and neuroprotection. Mol Neurobiol 2005;32:89-103.

7. Bazan NG. Synaptic lipid signaling: Significance of polyunsaturated fatty acids and platelet-activating factor. J Lipid Res 2003;44:2221-33.

8. Kato K, Clark GD, Bazan NG, Zorumski CF. Platelet-activating factor as a potential retrograde messenger in CA1 hippocampal long-term potentiation. Nature 1994;367:175-9.

9. Belayev L, Eady TN, Khoutorova L, Atkins KD, Obenaus A, Cordoba M, et al. Superior neuroprotective efficacy of LAU-0901, a novel platelet-activating factor antagonist, in experimental stroke. Transl Stroke Res 2012;3:154-63.

10. Belayev L, Khoutorova L, Atkins K, Gordon WC, Alvarez-Builla J, Bazan NG. LAU-0901, a novel platelet-activating factor antagonist, is highly neuroprotective in cerebral ischemia. Exp Neurol 2008;214:253-8.

11. Belayev L, Khoutorova L, Atkins K, Cherqui A, Alvarez-Builla J, Bazan NG. LAU-0901, a novel platelet-activating factor receptor antagonist, confers enduring neuroprotection in experimental focal cerebral ischemia in the rat. Brain Res 2009;1253:184-90.

12. Bazan NG. Neuroprotectin D1-mediated anti-inflammatory and survival signaling in stroke, retinal degenerations, and Alzheimer's disease. J Lipid Res 2009;50 Suppl: S400-5.

13. Bazan NG, Eady TN, Khoutorova L, Atkins KD, Hong S, Lu Y, et al. Novel aspirin-triggered neuroprotectin D1 attenuates cerebral ischemic injury after experimental stroke. Exp Neurol 2012;236:122-30.

14. Serhan CN, Fredman G, Yang R, Karamnov S, Belayev LS, Bazan NG, et al. Novel proresolving aspirin-triggered DHA pathway. Chem Biol 2011;18:976-87.

15. Eady TN, Khoutorova L, Anzola DV, Hong SH, Obenaus A, Mohd-Yusof A, et al. Acute treatment with docosahexaenoic acid complexed to albumin reduces injury after a permanent focal cerebral ischemia in rats. PLoS One 2013;8:e77237.

16. Belayev L, Alonso OF, Busto R, Zhao W, Ginsberg MD. Middle cerebral artery occlusion in the rat by intraluminal suture. Neurological and pathological evaluation of an improved model. Stroke 1996;27:1616-22.

17. Ghosh N, Yuan X, Turenius CI, Tone B, Ambadipudi K, Snyder EY, et al. Automated core-penumbra quantification in neonatal ischemic brain injury. J Cereb Blood Flow Metab 2012;32:2161-70.

18. Tian X, Bazan NG. Neuroprotection by platelet-activating factor antagonism. Ann N Y Acad Sci 2005;1053:455-6.

19. Belayev L, Mukherjee PK, Balaszczuk V, Calandria JM, Obenaus A, Khoutorova L, et al. Neuroprotectin D1 upregulates Iduna expression and provides protection in cellular uncompensated oxidative stress and in experimental ischemic stroke. Cell Death Differ 2017;24:1091-9.

20. Asatryan A, Bazan NG. Molecular mechanisms of signaling via the docosanoid neuroprotectin D1 for cellular homeostasis and neuroprotection. J Biol Chem 2017;292:12390-7.

21. Marcheselli VL, Hong S, Lukiw WJ, Tian XH, Gronert K, Musto A, et al. Novel docosanoids inhibit brain ischemia-reperfusion-mediated leukocyte infiltration and pro-inflammatory gene expression. J Biol Chem 2003;278:43807-17.

22. Eady TN, Belayev L, Khoutorova L, Atkins KD, Zhang C, Bazan NG. Docosahexaenoic acid signaling modulates cell survival in experimental ischemic stroke penumbra and initiates long-term repair in young and aged rats. PLoS One 2012;7:e46151.

23. Serhan CN, Dalli J, Colas RA, Winkler JW, Chiang N. Protectins and maresins: New pro-resolving families of mediators in acute inflammation and resolution bioactive metabolome. Biochim Biophys Acta 2015;1851:397-413.

24. Michael S. K, Stobo JD, Goldyne ME. In vitro synthesis of prostaglandins and related lipids by populations of human peripheral blood mononuclear cells. Prostaglandins 1980;20:135-45.

25. Nowak J, Kaijser L, Wennmalm A. Cardiac synthesis of prostaglandins from arachidonic acid in man. Prostaglandins Med 1980;4:205-14.

26. Riva M, Pappadà GB, Papadakis M, Cuccione E, Carone D, Menendez VR, et al. Hemodynamic monitoring of intracranial collateral flow predicts tissue and functional outcome in experimental ischemic stroke. Exp Neurol 2012;233:815-20.

27. Saleem S, Zhuang H, de Brum-Fernandes AJ, Maruyama T, Narumiya S, Doré S. PGD (2) DP1 receptor protects brain from ischemia-reperfusion injury. Eur J Neurosci 2007;26:73-8.

28. McCullough L, Wu L, Haughey N, Liang X, Hand T, Wang Q, et al. Neuroprotective function of the PGE2 EP2 receptor in cerebral ischemia. J Neurosci 2004;24:257-68.

29. Ahmad AS, Ahmad M, Maruyama T, Narumiya S, Doré S. Prostaglandin D2 DP1 receptor is beneficial in ischemic stroke and in acute exicitotoxicity in young and old mice. Age (Dordr) 2010;32:271-82.

30. Yang C, DeMars KM, Alexander JC, Febo M, Candelario-Jalil E. Sustained neurological recovery after stroke in aged rats treated with a novel prostacyclin analog. Stroke 2017;48:1948-56.

31. Hennebelle M, Zhang Z, Metherel AH, Kitson AP, Otoki Y, Richardson CE, et al. Linoleic acid participates in the response to ischemic brain injury through oxidized metabolites that regulate neurotransmission. Sci Rep 2017;7:4342.

32. Vangaveti VN, Jansen H, Kennedy RL, Malabu UH. Hydroxyoctadecadienoic acids: Oxidised derivatives of linoleic acid and their role in inflammation associated with metabolic syndrome and cancer. Eur J Pharmacol 2016;785:70-6.

33. Yigitkanli K, Pekcec A, Karatas H, Pallast S, Mandeville E, Joshi N, et al. Inhibition of 12/15-lipoxygenase as therapeutic strategy to treat stroke. Ann Neurol 2013;73:129-35. 